\title{
PHASE NOISE COMPENSATION FOR OFDM WLAN SYSTEMS USING SUPERIMPOSED PILOTS
}

\author{
Angiras R. Varma ${ }^{\dagger}$, Chandra R. N. Athaudage ${ }^{\dagger}$, Lachlan L.H Andrew ${ }^{\ddagger}$, Jonathan H. Manton* \\ ${ }^{\dagger}$ ARC Special Research Center for Ultra-Broadband Information Networks (CUBIN), University of Melbourne, Australia \\ * Research School of Information Sciences and Engineering, Australian National University, Canberra, Australia \\ ${ }^{\ddagger}$ Department of Computer Science, California Institute of Technology, California, USA
}

\begin{abstract}
In this paper, a novel phase-error estimation technique for OFDM based WLAN system is proposed. The proposed technique utilizes superimposed (or embedded) pilots for phase-tracking within OFDM data packet. This leads to saving transmission bandwidth, which is lost in the conventional phase-error estimation schemes with dedicated pilot subcarriers. Moreover, we propose selective pilot mapping and decision feedback as different strategies of improving the performance of the proposed technique. Simulation results confirm that the proposed technique has a similar symbol error performance to that of conventional scheme, however with the advantage of data-rate increase.
\end{abstract}

\section{INTRODUCTION}

Orthogonal frequency division multiplexing (OFDM) has been adopted for many WLAN standards such as $802.11 \mathrm{a} / 11 \mathrm{~g}$ and $802.11 \mathrm{n}$. In general WLAN standards are designed for the low mobility environment and therefore the channel estimation is performed using pilot OFDM symbols included in the preamble of each data packet. Channel equalization is performed for all OFDM data symbols contained in the packet using the estimated channel based on the received preamble. Given that the frequency-selective channel is time-invariant within a packet, the above channel equalization method works properly. However, Wiener phase noise (WPN) generated by the receiver RF oscillator is not time-invariant across the whole data packet. WPN causes (i) common phase rotation of post-FFT data (ii) inter-carrier interference (ICI) noise in post-FFT data and above (i) and (ii) varies from symbol-to-symbol within the same WLAN data packet. It should be noted that the common phase-rotation (constellation rotation) has a worse effect than ICI in terms of receiver error performance degradation. In WLAN standards (eg. 802.11a), a few dedicated pilot subcarriers running through the whole data packet (all OFDM blocks) is used to track the common phase-rotation caused by WPN. However, this causes bandwidth loss as pilot subcarriers are not available for data transmission (eg. $7.7 \%$ loss in 802.11a). A number of algorithms for phase noise estimation and suppression are presented in the literature $[1,2,3,4]$ using pilot subcarriers.

An alternate pilot allocation scheme is discussed in the literature known as superimposed or embedded pilot scheme. In the superimposed pilot scheme pilot symbols and information symbols are superimposed or arithmetically added before modulation and transmission. The advantage of this scheme is the avoidance of dedicated subcarriers/time-slots for pilots; thus saving the band- width. Superimposed scheme has been reported in the literature for channel estimation $[5,6,7,8]$.

In contrast to the dedicated pilot subcarriers, in this paper we propose using superimposed (embedded) pilots for tracking the common phase-rotation, thus saving the bandwidth for the pilot subcarriers. Also we propose "selective mapping" of superimposed pilots at the transmitter as a strategy of improving the phase estimation accuracy with superimposed pilots. Simulation results show that similar receiver error performance to standard pilot subcarriers can be achieved with superimposed pilots, but without the bandwidth loss due to pilot subcarriers.

\section{SYSTEM MODEL}

\subsection{Phase Noise Model}

The phase noise for the $n$th sample of the $m$ th OFDM symbol is modelled by a Wiener process as

$$
\phi_{n}(m)=\phi_{N-1}(m-1)+\sum_{i=0}^{N_{g}+n} \theta_{i}(m), 0 \leq n \leq N-1
$$

where $N_{g}$ is the cyclic prefix length, $N$ is the number of subcarriers and $\theta_{i}$ is a Gaussian random variable with mean zero and variance $\sigma_{\theta}^{2}[2]$.

\subsection{Signal Model}

The OFDM symbol structure for a WLAN system is given in Fig. 1. A preamble consisting of pilot OFDM blocks is transmitted for channel estimation and time/frequency offset estimation. After the preambles of the data packets, four subcarriers are dedicated for entire transmission time to estimate the common phase-rotation error. These dedicated subcarriers transmit pilot symbols to assist the phase noise estimation, whereas the remaining subcarriers carries information symbols. In contrast to the scheme of pilot allocation using dedicated subcarriers, this paper proposes an alternate scheme as depicted in Fig 2. It shares the preamble structure with the previous scheme, however all other subcarriers transmit both information and pilot symbols together in a superimposed fashion. The obvious advantage of the proposed scheme is saving of bandwidth for dedicated pilot subcarriers. In other words data rate of the system can be improved using the saved pilot subcarriers for data transmission. The basic signal model for the proposed scheme is discussed next. 
The data carried by the $k$ th subcarrier of an OFDM symbol is

$$
X_{k}=C_{k}+P_{k}
$$

where $C_{k}$ is the information symbol with variance $\sigma_{C}^{2}$ and $P_{k}$ is the superimposed pilot symbol with variance $\sigma_{P}^{2}$. Define $\eta=$ $\sigma_{C}^{2} /\left(\sigma_{C}^{2}+\sigma_{P}^{2}\right)$ is the ratio of information symbol power to total transmitted symbol power. In the superimposed pilot scheme, the power ratio $\eta$ can take values $0<\eta<1$, whereas in a conventional scheme $\eta=1$ when information symbols are transmitted $\left(X_{k}=C_{k}\right)$ and $\eta=0$ for pilot transmission $\left(X_{k}=P_{k}\right)$.

Consider a frequency-selective channel with memory $L$, and channel tap value vector $\boldsymbol{h}=\left[h_{0} \ldots h_{L-1}\right]$. The received OFDM sample $y_{n}$ is given by

$$
y_{n}=\sum_{l=0}^{L-1} h_{l} x_{n-l} e^{j \phi_{n}}+w_{n}
$$

where $\phi_{n}$ is the time-domain phase error due to WPN introduced at the receiver and $w_{n}$ is the channel noise which is Gaussian distributed $\mathcal{N}\left(0, \sigma_{w}^{2}\right)$. In (3), $\boldsymbol{x}=\left[x_{0} x_{1} \ldots x_{N-1}\right]$ is the IFFT of the data symbol $\boldsymbol{X}=\left[X_{0} X_{1} \ldots X_{N-1}\right]$. The post-FFT signal at the receiver (FFT of $y_{n}, 0 \leq n \leq N-1$ ) is

$$
Y_{k}=H_{k} X_{k} S_{0}+\sum_{l=0}^{N-1} H_{l} X_{l} S_{l-k}+W_{k}
$$

where $H_{k}$ and $S_{l}$ are the channel frequency response and inter-

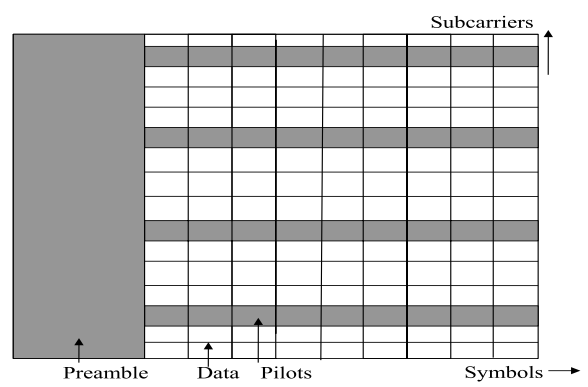

Fig. 1. Conventional pilot structure for OFDM based WLAN system.

carrier interference (ICI), respectively. The ICI term $S_{l}$ is a function of the phase noise $\phi_{n}$ given by

$$
S_{l}=\frac{1}{N} \sum_{n=0}^{N-1} e^{j 2 \pi n l / N} e^{\phi_{n}}, l=0, \ldots, N-1 .
$$

From (4), it can be seen that the phase noise causes common phase error as well as ICI. The received post-FFT signal given in (4) can be written as

$$
Y_{k}=H_{k} C_{k} S_{0}+H_{k} P_{k} S_{0}+I_{k}+W_{k}
$$

where $I_{k}$ is the ICI term (second term of (4)). The effect of $S_{0}$ on the post-FFT data symbols $C_{k}^{\prime} \mathrm{s}$ is a common-phase-rotation.

Since the preambles are used for the channel estimation at the beginning of each packet, channel information is available for phase estimation. The task is then as follows: Given $Y_{k}, H_{k}$ and $P_{k}$ for all $0 \leq k \leq N-1$, estimate $S_{0}$.

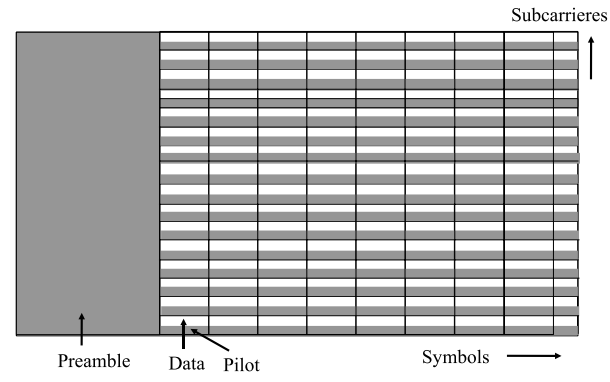

Fig. 2. Superimposed pilot scheme for OFDM based WLAN system.

\section{PROPOSED PHASE ROTATION ESTIMATION}

This section introduces the least squares estimator for phase estimation and the selective mapping of pilots for performance enhancement.

\subsection{The Least Squares Estimation with Averaging}

The least squares estimation with averaging scheme treats the contribution of the unknown information symbol $C_{k}$ in the received signal (post-FFT) $Y_{k}$ as noise. This means that the term $H_{k} C_{k} S_{0}$ is a noise term in (6). Thus, $Y_{k}$ can be expressed as

$$
Y_{k}=H_{k} P_{k} S_{0}+Z_{k}
$$

where $Z_{k}=H_{k} C_{k} S_{0}+I_{k}+W_{k}$ is the total noise. The least squares (LS) estimate of the phase-rotation term $S_{0}$ based on $k$ th subcarrier signal is

$$
\hat{S}_{0}(k)=\frac{Y_{k}}{H_{k} P_{k}} .
$$

Substitution of (6) in (8) gives

$$
\hat{S_{0}}(k)=S_{0}+\frac{S_{0} C_{k}}{P_{k}}+\frac{V_{k}}{H_{k} P_{k}}
$$

where $V_{k}=I_{k}+W_{k}$. In (9), $\hat{S}_{0}(k)$ is the initial estimate obtained only using $k$ th post-FFT signal. However this estimate can be improved as follows.

In a frequency selective channel, different subcarriers experience different fading according to the channel conditions. In the conventional techniques of phase estimation, if a dedicated pilot subcarrier falls in deep fade, the phase estimation accuracy would be adversely affected. However, in superimposed pilot scheme since pilots are present in all the subcarriers, it is advantageous to use subcarriers that have better channel response for phase estimation instead of using all the subcarriers. This can be effectively implemented as the channel state information is present at the receiver (Since the preamble can be used to estimate the channel). Thus we can use subcarrier selection for phase estimation as follows. Compute $\boldsymbol{\Omega}=\left\{\left|H_{i}\right|^{2} \mid, 0 \leq i \leq N-1\right\}$ and select set of indices $\mathbf{I}=\left\{k_{0}, k_{1}, \ldots, k_{N_{0}-1}\right\}$ corresponding to the $N_{0}$ highest elements of $\boldsymbol{\Omega}$.

Some assumptions about the noise terms in (9) can be made in the presence of above mentioned subcarrier selection. The second and the third terms in (9) are noise terms and it is valid to 


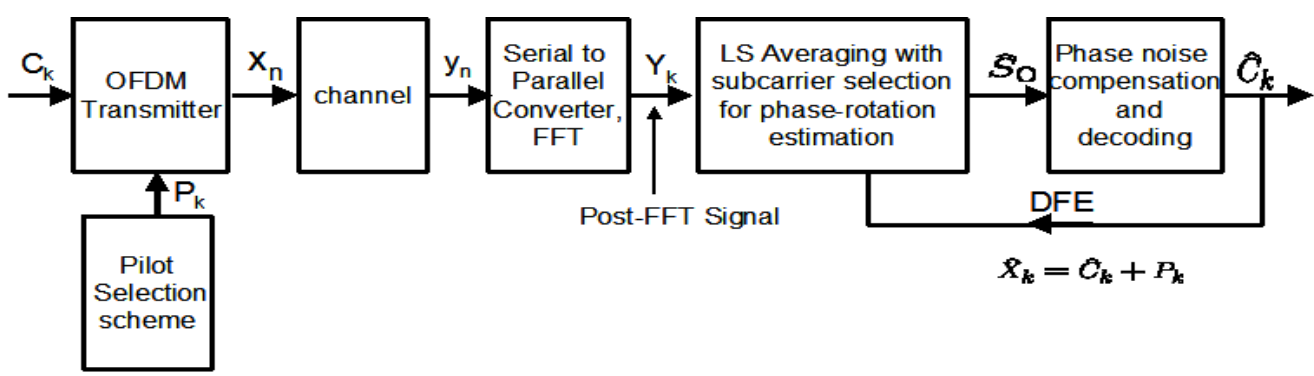

Fig. 3. The OFDM transmission system incorporating the proposed phase-error estimation technique.

assume that the variance of third term in (9), $\frac{V_{k}}{H_{k} P_{k}}$ is negligible compared to the variance of the second term $\frac{S_{0} C_{k}}{P_{k}}$ due to following reasons. $(i)$ With the subcarrier selection the lower values of $\left|H_{k}\right|^{2}$ are eliminated and $(i i)$ the variance of the transmitted symbols $C_{k}$, which is contributing towards the noise term, is higher than the sum of variances of the ICI term and channel noise, $V_{k}$. With this assumption, it can be noted that the variance of the noise term in (9) is approximately constant irrespective of channel and the subcarrier. Since variance of the noise terms is constant over the subcarriers, an eqaul weight averaging scheme is proposed to improve the estimate of $S_{0}$ as

$$
\hat{S}=\frac{1}{N_{0}} \sum_{k \in \boldsymbol{I}} \hat{S}_{0}(k)
$$

Substituting for $\hat{S_{0}}(k)$ in (10) gives

$$
\begin{aligned}
\hat{S_{0}} & =S_{0}+\frac{1}{N_{0}} \sum_{k \in \boldsymbol{I}} \frac{S_{0} C_{k}}{P_{k}}+\frac{1}{N_{0}} \sum_{k \in \boldsymbol{I}} \frac{V_{k}}{H_{k} P_{k}} \\
& =S_{0}+S_{0} \alpha+\beta
\end{aligned}
$$

where $\alpha=\frac{1}{N_{0}} \sum_{k \in \boldsymbol{I}} \frac{C_{k}}{P_{k}}, \beta=\frac{1}{N_{0}} \sum_{k \in \boldsymbol{I}} \frac{V_{k}}{H_{k} P_{k}}$ and $S \alpha+\beta$ denotes the total estimation error. The maximum ratio combining(MRC) is a simple alternative, which should give better performance than selection diversity. The proposed technique is shown as part of the block diagram in Fig. 3. Performance results are presented in Section 5.

\subsection{Selective Mapping of Pilots}

The phase noise estimation scheme described in subsection 3.1 treats the contribution of the information symbols in the received symbols as noise. The noise from the information symbols is expressed in the phase noise estimation error expression (12) as $S_{0} \alpha$. Thus one approach of reducing this noise is to make $\alpha$ as small as possible. This can be achieved by reducing the correlation between the information symbols and pilots or by reducing $\sum_{k=0}^{N-1} P_{k}^{*} C_{k}$. To this end we introduce a random codebook of pilot symbols at the transmitter and a particular pilot sequence is selected from the codebook such that the noise due to information symbols $\alpha$ is minimized. The pilot mapping is done as follows:

- Define a random codebook of pilots

$$
\mathcal{B}=\left\{P_{0}^{j}, \ldots, P_{N-1}^{j}\right\}, \text { where } j \in \mathcal{I}_{\mathcal{B}}
$$

where $\mathcal{B}$ is the codebook of pilots and $\mathcal{I}_{\mathcal{B}}=\{0,1, \ldots, M-$ $1\}$ is the set of codebook indices.
- Find the codebook index that minimizes $\alpha$ as

$$
\tilde{i}=\arg \min _{i \in \mathcal{I}_{\mathcal{B}}} \sum_{k=0}^{N-1} P_{k}^{i *} C_{k} .
$$

- Transmitted signal sequence with the pilot mapping is

$$
X_{k}=C_{k}+P_{k}^{\tilde{i}}, 0 \leq k \leq N-1 .
$$

The information about the selected pilot sequence is informed to the receiver either by sending the index of the codebook or by evaluating the correlation of received symbols with all entries in the codebook. The former option demands an overhead of $\log _{2} M$ bit per OFDM symbol, where as the latter option increases complexity of the receiver. Although the former option introduces overhead, still this scheme is advantageous in terms of bandwidth saving for higher order modulation schemes like QPSK or more. Performance of the proposed scheme is demonstrated in Section 5. The proposed technique is presented as "Pilot selection scheme" in Fig. 3.

\section{PHASE ERROR COMPENSATION AND SYMBOL DECODING}

The schemes for phase-error compensation and symbol decoding are presented in this section. The phase estimate obtained in Section 3 is used to compensate for the phase-rotation and then the transmitted symbols are decoded. To improve the performance of the proposed scheme a decision feedback scheme is also proposed. The proposed schemes are presented as a block diagram in Fig. 3.

\subsection{Symbol Decoding}

The contribution of pilot symbols in the received symbol $Y_{k}$ is removed as

$$
Y_{k}^{\prime}=Y_{k}-\hat{S_{0}} H_{k} P_{k} .
$$

Substituting for $Y_{k}$ in (16) from (7) gives

$$
Y_{k}^{\prime}=S_{0} H_{k} C_{k}-\left(\alpha S_{0}+\beta\right) H_{k} P_{k}+V_{k}
$$

A decision variable can be obtained from (17) as

$$
\tilde{C}_{k}=\frac{Y_{k}^{\prime}}{\hat{S_{0} H_{k}}}
$$


$\tilde{C}_{k}$ is further expanded with the noise terms as

$$
\begin{aligned}
\tilde{C}_{k} & =C_{k}-\left[\frac{\alpha S_{0}+\beta}{S_{0}(1+\alpha)+\beta}\right] C_{k}+\left[\frac{\alpha S_{0}+\beta}{S_{0}(1+\alpha)+\beta}\right] P_{k} \\
& +\frac{V_{k}}{\left[S_{0}(1+\alpha)+\beta\right] H_{k}} .
\end{aligned}
$$

In (19), the second term is the ISI (inter-symbol interference), third term is pilot interference and last term is the AWGN interference. The transmitted symbol can be decoded using nearest neighbor demapping, $\hat{C}_{k}=Q\left(\tilde{C}_{k}\right)$.

\subsection{Decision Feedback}

The estimated information symbol can be used for re-estimation of the common phase error. The estimate of the transmitted symbol can be obtained from the estimated information symbol as $\hat{X}_{k}=$ $\hat{C}_{k}+P_{k}$ and the estimate of the common phase error is obtained as

$$
\breve{S}_{0}(k)=\frac{Y_{k}}{H_{k} \hat{X}_{k}}
$$

and

$$
\breve{S}_{0}=\frac{1}{N_{0}} \sum_{k \in \boldsymbol{I}} \breve{S}_{0}(k)
$$

Note that we only use the best subcarriers for the decision feedback to improve the performance. Now $\breve{S}_{0}$ can be used instead $\hat{S}_{0}$ in the symbol decoding scheme in Section 4.1. subsectionPower Allocation In (2), as we vary the power allocation factor $\eta$ the proportion of information symbol and pilot symbol vary in the transmitted symbol. As the proportion of pilot power increases in the transmitted symbol, the common phase error estimation accuracy would increase, however the received information symbols SNR would decrease adversely affecting the SER performance of the receiver. Thus it is important to find the optimal ratio of pilot power to total power that would minimize the SER. A simulation study is done to find the optimal power ratio and the results are presented in Section 5.

\section{NUMERICAL RESULT}

This section provides the simulation results for the schemes described in the previous sections. We consider a WLAN system with 64 subcarriers of which 8 subcarriers are null subcarriers and 52 are transmitting information symbols. Cyclic-prefix length is 16 samples. A Rayleigh-fading channel is modelled as a finite impulse response filter with tap length of 10 . A uniform power delay profile of the channel is considered as a worst case scenario. Phase error is generated using (1) and it is applied to the received OFDM symbols in time domain. The channel noise is AWGN and the SNR range $0-20 \mathrm{~dB}$.

\subsection{Conventional Scheme for Comparison}

We consider the following scheme based on frequency-multiplexed pilots for comparison with our proposed scheme [9]. In this scheme $N_{0}=4$ subcarriers are allocated with the pilot symbols and are used for common phase error estimation. Let $\mathcal{P}$ be the set of indices of pilot subcarriers. In the conventional scheme $\eta=1$ so

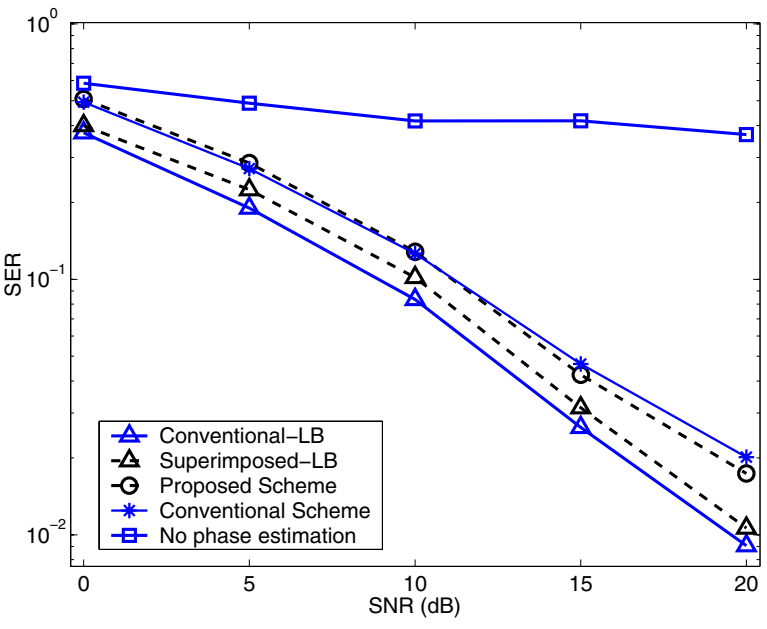

Fig. 4. SER performance of the superimposed scheme with the conventional scheme for QPSK modulation.

$X_{k}=P_{k}$ from $k \in \mathcal{P}$. Post-FFT signal model in the receiver as represented in (6) becomes

$$
Y_{k}=H_{k} P_{k} S_{0}+Z_{k}, k \in \mathcal{P}
$$

where $Z_{k}=I_{k}+W_{k}$, sum of the ICI noise and the channel noise. There is no contribution of noise from the information symbols. The phase estimation can be done as

$$
\hat{S}=\frac{1}{N_{0}} \sum_{k \in \mathcal{P}} \frac{Y_{k}}{H_{k} P_{k}}
$$

and the decision variable for symbol decoding is

$$
\tilde{C}_{k}=\frac{Y_{k}}{\hat{S} H_{k}} .
$$

Symbol decoding can be done as $\hat{C_{k}}=Q\left(\tilde{C_{k}}\right)$ using the nearest neighbor mapping. The performance comparison of the proposed scheme with the conventional scheme is given in the next subsection.

\subsection{SER Comparison}

The SER performance comparison of the conventional scheme and the proposed schemes are given in the Fig 4, 5. These performance results are for QPSK and BPSK modulation schemes. The variance of the phase noise random variable $\theta$ in (1) is $\sigma_{\theta}^{2}=$ 0.49 square degree. The subcarrier selection uses $N_{0}=48$ best subcarriers for phase noise estimation. Phase mapping for proposed superimposed pilot scheme uses $M=16$ codebook entries and decision feedback.

Without any phase estimation error the SER curves are plotted for both the conventional scheme (CONV:LB) and the superimposed scheme (SUP:LB). These two curves are the lower bounds of performances. The lower bound of the superimposed scheme is slightly worse than that of the conventional scheme since a proportion of the transmitted signal power is allocated for the superimposed pilots reducing the effective SNR. The upper bound of 


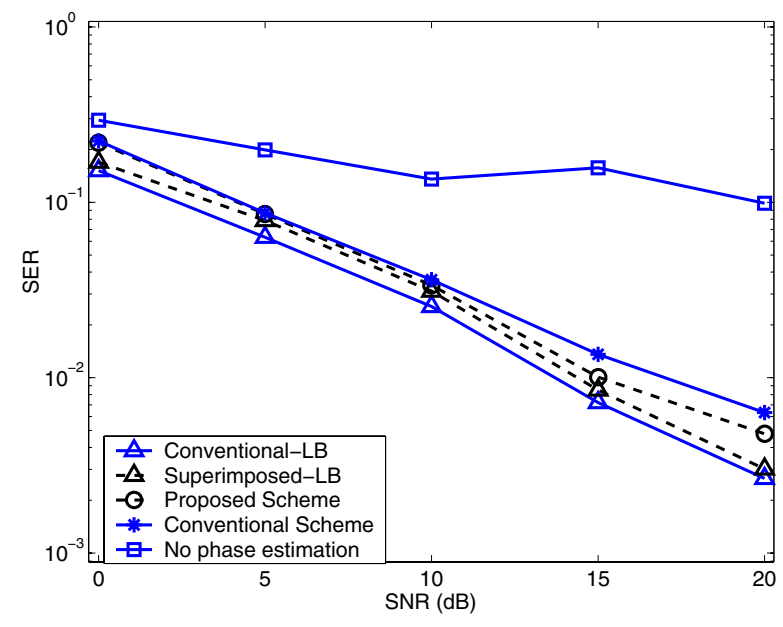

Fig. 5. SER performance of the superimposed scheme with the conventional scheme for BPSK modulation.

performances is obtained by decoding the received symbols without phase estimation and is denoted in the graph as "No phase estimation".

The proposed scheme performs as well as the conventional scheme. However the proposed scheme has the advantage in terms of saving of bandwidth for dedicated pilot subcarriers. Overall data rate of the proposed scheme would be more than the conventional scheme since this extra bandwidth is used for the data transmission.

\subsection{Performance of power allocation scheme}

SER is determined for different pilot power ratios $1-\eta$. The resulting graphs is given in Fig 6. SER decreases as the pilot power ratio increase to some extent and then SER start increasing. The optimal pilot power ratio is obtained as $4 \%, 6 \%$ at $20 \mathrm{~dB}$ and 10 $\mathrm{dB}$ channel SNR respectively. Similarly optimal power ratios are obtained as $8 \%, 6 \%, 4 \%$ for channel SNR $0 \mathrm{~dB}, 5 \mathrm{~dB}$ and $15 \mathrm{~dB}$ respectively. Optimal power ratios are used in the simulations in previous subsection.

\section{CONCLUSION}

A novel phase-error estimation scheme is proposed for OFDM based WLANs. The proposed scheme uses superimposed pilots for phase noise estimation, effectively saving the bandwidth for dedicated pilot subcariers. Simulation results demonstrate that the proposed phase-error estimation scheme performs as well as the conventional scheme.

\section{Acknowledgment}

This work was supported by the Australian Research Council.

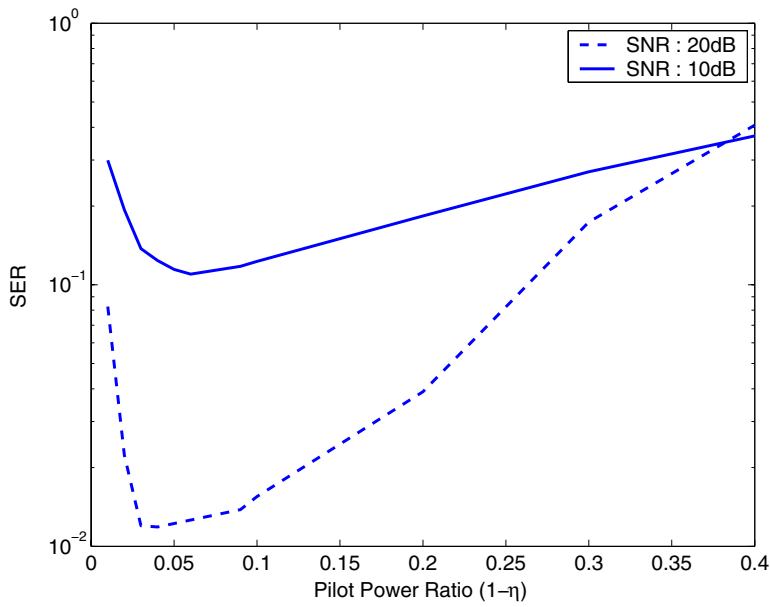

Fig. 6. SER performance as a function of pilot power ratio $(1-\eta)$.

\section{REFERENCES}

[1] S. Wu and Y.Bar-Ness, "A phase noise suppression algorithm for OFDM-based WLANs," IEEE Transactions on Communication Letters, vol. 6, no. 12, pp. 535 - 537, 2002.

[2] K. Nikitopoulos and A. Polydoros, "Compensation schemes for phase noise and residual frequency offset in OFDM systems," in Proc. Globcom 2001, Nov. 2001, vol. 1, pp. 330 333.

[3] P. Robertson and S. Kaiser, "Analysis of the effects of phasenoise in orthogonal frequency division multiplex (OFDM) systems," in Proc. ICC 1995, June 1995, vol. 3, pp. 1652 1657.

[4] T. Pollet, M. Van Bladel, and M. Moeneclaey, "BER sensitivity of OFDM systems to carrier frequency offset and Wiener phase noise," IEEE Transactions on Communication, vol. 43, no. 234, pp. 191-193, 1995.

[5] B. Farhang-Boroujeny, "Pilot-based channel identification: proposal for semi-blind identification of communication channels," Electronics Letters, vol. 31, no. 13, pp. 1044 - 1046 , June 1995.

[6] J. H. Manton, I. M. Y. Mareels, and Y. Hua, "Affine precoders for reliable communications," in Proc. IEEE ICASSP, Istanbul, Turkey, June 2000, vol. V, pp. 2749-2752.

[7] J. K. Tugnait and X. Meng, "MIMO channel estimation using superimposed training," in Proceedings of IEEE International Conference on Communications, June 2004, vol. 5, pp. 2663 2667.

[8] A.R. Varma, C. Athaudage, L.L.H Andrew, and J.H. Manton, "Optimal superimposed pilot training for OFDM channel estimation," in Proc. SPAWC 2006, July 2006.

[9] J. Heiskala and J. Terry, OFDM Wireless LANS: A Theroetical and Practical Guide, SAMS, first edition, 2002. 